\title{
Tubulovillous adenoma of anal canal
}

\author{
Ahmed Abudames and Awad Ali Mohamed Ahmed Alawad* \\ Department of Surgery, Prince Sultan Armed Forces Hospital, Medina, Saudi Arabia
}

A 78 year-old man was referred to our outpatient clinic with history of rectal bleeding for several months. Bleeding occurred with almost every bowel movement and consisted of passage of fresh blood. The patient was otherwise healthy with no recent change in bowel habit, weight loss or reduced appetite. The patient was referred to the gastrointestinal service for work-up of rectal bleeding. His past history was positive only for hypertension. General physical examination was unremarkable. At the time of colonoscopy, rectal examination revealed a $6 \mathrm{~cm}$ friable mass attached to the anus by a definite stalk (Figure 1A). The mass was located entirely outside the anal canal, with no extension into the rectum. Colonoscopy showed no synchronous polyps. Biopsy was taken and revealed tubulovillus adenoma. CT scan showed an anal mass with no invasion or lymphadenopathy (Figure 1B).

At surgery, the anal polyp was found to be attached to the anus by a stalk. The mass was grasped with a Babcock forceps and was resected using harmonic scalpel. Histopathologic examination revealed a tubulovillus adenoma (TVA). There were no areas of high
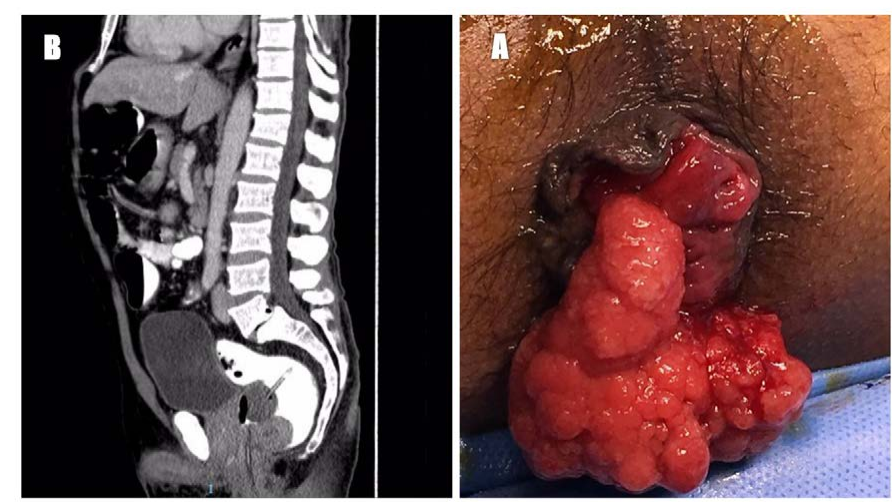

Figure 1. (A) Image shows anal polyp, found to be attached to the anus by a stalk (B) CT scan shows anal mass.

Copyright: (C2017 Abudames A. This is an open-access article distributed under the terms of the Creative Commons Attribution License, which permits unrestricted use, distribution, and reproduction in any medium, provided the original author and source are credited. grade dysplasia or malignant transformation. The squamocolumnar junction was visible at the edges of the lesion and did not show any atypical changes. We believe the site of origin of the tubulovillus adenoma in our patient was from one of the anal glands which are the only adenomatous elements in the anus. It is unclear why such tumors are not diagnosed more frequently. It is possible that because of the submucosal location of the anal glands, such adenomas are not easily visible and only become apparent when malignant transformation takes place $[1,2]$. Clinically, it is important to recognize and treat these tumors at an early stage because of their potential to transform into adenocarcinoma.

\section{References}

1. Malassagne B, Penna C, Parc R (1995) Adenomatous polyps in the anal transitional zone after ileal pouch-anal anastomosis for familial adenomatous polyposis: treatment by transanalmucosectomy and ileal pouch advancement. Br J Surg 82: 1634. [Crossref]

2. Corman M, Allison SI, Kuehne J (2002) Malignant tumors of the anal canal. In Handbook of Colon \& Rectal Surgery. Philadelphia: Lippincott Williams \&Wilkins; USA.

Correspondence to: Awad Ali Mohamed Ahmed Alawad, Department of Surgery, Prince Sultan Armed Forces Hospital, Medina, Saudi Arabia, E-mail: awadali82@hotmail.com

Received: January 15, 2017; Accepted: January 26, 2017; Published: January 29,2017 